\title{
Compliance and psychological reactance in schizophrenia
}

\author{
Antonia Moore* \\ Department of Psychology and Speech Pathology, Manchester Metropolitan University, UK \\ William Sellwood \\ Department of Clinical Psychology, University Hospital of South Manchester, UK

\begin{abstract}
John Stirling
Department of Psychology and Speech Pathology, Manchester Metropolitan University, UK
\end{abstract}

Objectives. Non-compliance with neuroleptic medication in schizophrenia is a major cause of relapse. A number of sociodemographic variables, and illness, attitudinal and treatment variables, have been demonstrated to be associated with non-compliance. The present study examined a range of these variables and their predictive value in determining past and current compliance.

Methods. Thirty-nine patients suffering from schizophrenia and three patients suffering from schizoaffective disorder completed a series of questionnaires assessing psychological reactance, insight, subjective response to medication, perceived threat to freedom of choice, and degree of current and past compliance. Logistic regression analyses were performed to determine which factors best predicted past and current compliance.

Results. Psychological reactance and age were found to be the best predictors of past compliance, with an interaction between reactance and perceived threat to freedom of choice posed by treatment provision also making a significant contribution. Past compliance behaviour and subjective response to medication predicted current compliance most significantly.

Conclusions. Reactance is an important predictor of compliance history especially when patients perceive treatment to be a threat to freedom of choice. Subjective response to neuroleptics is most important in predicting current compliance. Implications for intervention are discussed.

Compliance with antipsychotic medication is crucial in the prevention of symptom aggravation and relapse in patients suffering from schizophrenia. It has been shown that over a 9-month period, approximately $50 \%$ of patients relapse when not

* Requests for reprints should be addressed to Antonia Moore, Department of Psychology and Speech Pathology, Manchester Metropolitan University, Elizabeth Gaskell Campus, Hathersage Road, Manchester M13 0JA, UK. 
regularly taking their medication (Bebbington \& Kuipers, 1994; Vaughn \& Leff, 1976).

Non-compliance has been found to be associated with greater psychopathy (Kasper, Hoge, Feucht-Haviar, Cortina, \& Cohen, 1997; Hoge et al., 1990), adverse side-effects to medication (Fleishhacker, Meisa, Gunther \& Kurz, 1994), a poor subjective response to medication (Awad, 1993), poor insight into illness (Ghaemi \& Pope, 1994), and negative patient attitudes (Hoge et al., 1990; Kelly, Mamon, \& Scott, 1987). Tunnicliffe, Harrison, and Standen (1992) and Zito, Routt, Mitchell and Roerig (1985) have suggested that being young and male are risk factors for noncompliance, but others do not report these observations (Buchanan, 1992; Baekeland \& Lundwall, 1975). Although associations between these factors and compliance have been demonstrated, they do not explain why individuals exhibit noncompliance.

A potential variable of interest within the area of non-compliance is psychological reactance, a motivational state that can develop when a person perceives that there is a threat to his or her personal freedom (Brehm, 1966; Brehm \& Brehm, 1981). Reactance is directed towards restoring the behaviour that is being threatened and can be interpreted as a direct reassertion of a freedom through oppositional behaviour. Individual differences in reactance level have been observed (Dowd, Milne, \& Wise, 1991), and Graybar, Antonuccio, Boutilier, \& Varble (1989) have suggested that highly reactant patients may perceive high amounts of clinician advice to be a threat to their freedom. Reactance prone individuals may therefore attempt to reassert this freedom through oppositional or non-compliant behaviour. If a relationship between reactance proneness and non-compliance could be demonstrated, then such an association could have clinical implications. If patients who are reactance prone perceive treatment provision to be a threat to their freedom of choice, treatment provision could be alerted in such a way so as to avoid inducing reactance, and thus non-compliance.

The present study attempted to examine the relationship between compliance and reactance in patients with schizophrenia. It was hypothesized that highly reactant patients would be more likely to be non-compliant with antipsychotic medication than patients with a low level of reactance, particularly if they perceived treatment to be a threat to their freedom of choice. The relationships between compliance and demographic, illness and treatment variables were also examined, in order to determine the extent to which these factors were related to compliance.

\section{Method}

\section{Participants}

Forty-two patients who had an ICD-9 diagnosis (World Health Organization, 1978) of 295.0 to 295.9 (schizophrenic disorders and schizoaffective disorder $(N=3)$ ) were recruited (ICD-9 diagnoses were recorded by patients' psychiatrists at discharge). All participants were out-patients who were either attending the local psychiatric day hospital or taking part in a larger scale study (Barrowclough et al., 1999) at the time of recruitment. The patients had all been prescribed anti-psychotic medication. Twenty-seven men and 15 women participated. Their mean age was 39 years $(\mathrm{SD}=11)$, with a range of 19-63 years. The majority of patients had left school at $16(N=33)$, were not married $(N=27)$, and received little family support $(N=26)$. The mean number of admissions was $5.6(\mathrm{SD}=4 \cdot 1)$, the 
mean duration of illness was 14.3 years $(S D=10 \cdot 1)$, and the mean number of days of last admission was $38 \cdot 3(\mathrm{SD}=39 \cdot 7)$.

\section{Procedure}

Symptoms were rated using the Krawiecka-Goldberg-Vaughan (KGV) scale (Krawiecka, Goldberg, \& Vaughan, 1977). This scale enables ratings to be made on a profile of eight symptoms which can be categorized into three groups-affective, positive and negative. The mean positive symptom score was $2.50(\mathrm{SD}=2.55)$, the mean negative symptom score was $2.19(\mathrm{SD}=2.24)$, the mean affect score was $2.45(\mathrm{SD}=1.97)$, and the mean total $\mathrm{KGV}$ score was $7.14(\mathrm{SD}=4.03)$. The patients were asked to complete a series of questionnaires. A number of the scales used were self-report questionnaires, however a researcher (A.M.) went through each questionnaire clarifying items at patients' requests. The psychiatric assessment and questionnaires usually took 1 hour to complete. Information concerning previous psychiatric history and prescription details was obtained from medical records.

\section{Compliance with antipsychotic medication}

The patients were asked to rate their current and past compliance using two 4-point scales that were developed for another study (Barrowclough et al., 1999). The current compliance measure yielded a rating based on medication-taking during the 9 months prior to the study. Patients were asked whether they had taken their medication at least $90 \%$ of the time, between $50 \%$ and $90 \%$ of the time, between $10 \%$ and $50 \%$ of the time, or less than $10 \%$ of the time during the past 9 months. This timeperiod was selected because non-compliance has been demonstrated to have a negative effect on outcome during a 9-month period (Vaughn \& Leff, 1976). The past compliance measure yielded a similar rating based on medication-taking from the time of initial diagnosis to 9 months prior to the present study. Individuals were classified as compliers if they took at least $90 \%$ of their prescribed medication, and non-compliers if they took less than $90 \%$ of their prescribed medication during the specified timeperiods for current and past compliance (Vaughn \& Leff, 1976).

\section{Other measures}

Psychological reactance was assessed using the Hong Psychological Reactance Scale (HPRS; Hong \& Page, 1989). This scale has demonstrated high reliability with a non-clinical population and exhibits a clearly defined 4-factor structure-freedom of choice, conformity reactance, behavioural freedom, and reactance to advice and recommendations. The degree of perceived threat to personal freedom from treatment provision was assessed using the Psychiatry Reactance Scale (PRS) consisting of seven generated items which were related to freedom of choice and treatment (see Appendix 1). Test-retest reliability of the HPRS with a clinical population, and the PRS, was established by asking a random selection of participants $(N=19)$ to repeat the questionnaires after an interval of 2 weeks. The test-retest reliability coefficient for the HPRS was 0•86, and for the PRS was 0.68.

Insight into illness was assessed by the Birchwood et al. Insight Scale (INS) (Birchwood et al., 1994). This scale incorporates three components-awareness of illness, relabelling of symptoms as pathological, and the perceived need for treatment-and has been demonstrated to be reliable, valid, and sensitive to change. Individuals' subjective experience of drug effects and beliefs about treatment was measured using the Drug Attitude Inventory (DAI) (Hogan, Awad, \& Eastwood, 1983). This inventory has been shown to be predictive of treatment compliance, both observer-rated and as measured by biological assay, and is reliable and valid.

\section{Results}

\section{Current compliance}

Non-compliant patients were significantly more likely to experience a poorer subjective response compared with compliant patients $(U=115 \cdot 5, p=.01)$, but they did not exhibit significantly more severe psychopathology, greater levels of 
Antonia Moore et al.

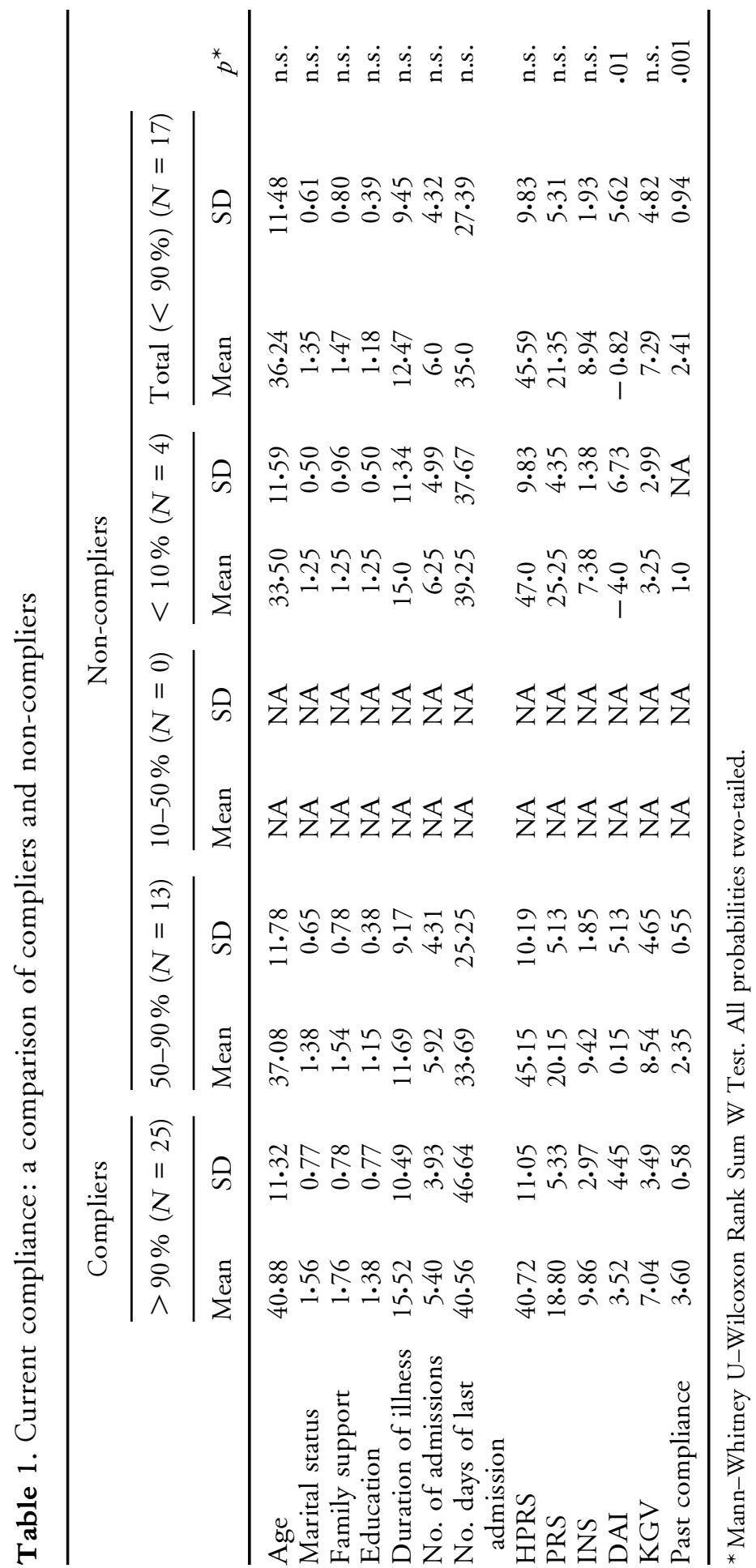




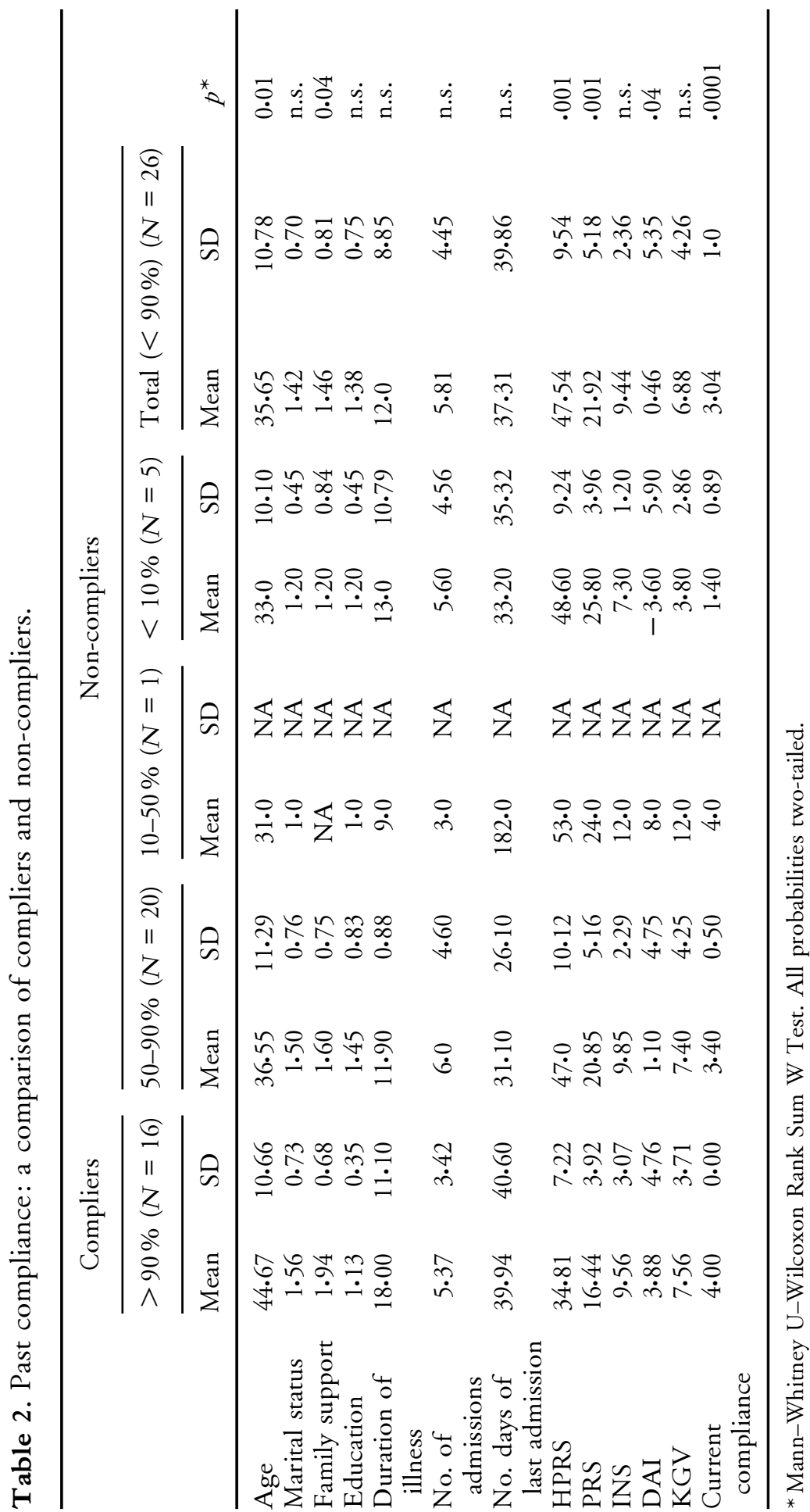


reactance, nor did they perceive treatment provision to be a greater threat to their personal freedom than did compliant patients (Table 1). Patients in both groups exhibited similar levels of insight, although a second analysis of insight with the need for treatment component removed revealed that non-compliant patients had less insight $(U=140 \cdot 0, p=.05)$. This secondary analysis was then performed to account for any confounding effects of the perceived need for treatment component on compliance. Compliant patients were more likely to have been compliant in the past compared with currently non-compliant patients $(U=60 \cdot 0, p=.001)$.

Significant correlations were observed between current compliance and the DAI $(r=0.42 ; p=.006)$, degree of insight $(r=0.32 ; p=.04)$, and past compliance $(r=$ $0.72 ; p=.001)$.

Logistic regression revealed that no demographic variables predicted current compliance. Past compliance was the best predictor of current compliance $(B(1)=$ $-2.84 ; p=.008)$, with the DAI also making a significant contribution $(B(1)=$ $-0.17, p=.01)$.

\section{Past compliance}

Non-compliant individuals did not exhibit significantly different levels of insight compared with compliant individuals nor did they exhibit a greater degree of psychopathology (Table 2). Individuals who had been non-compliant previously were less likely to receive any family support $(U=139.5 ; p=.04)$ and were younger $(U=103.5 ; p=.01)$ than those patients who had been compliant. Participants who had been non-compliant were found to exhibit more reactance $(U=66.0 ; p=.0002)$, and were more likely to feel that their personal freedom was being threatened by treatment provision $(U=86.5 ; p=.0016)$. They were also more likely to experience a poor subjective response as measured by the DAI $(U=131.0 ; p=.04)$. Patients who had been compliant in the past were more likely to be currently compliant compared with individuals who had been non-compliant in the past $(U=72 \cdot 0 ; p=$ $.0001)$.

Significant correlations were observed between past compliance and age $(r=.42$; $p=.006)$, the DAI $(r=.35 ; p=.02)$, degree of family support $(r=.38 ; p=.01)$, reactance $(r=-.56 ; p=.001)$, and the degree of perceived threat to freedom from treatment provision $(r=-0.56 ; p=.001)$.

Logistic regression revealed that reactance made the most significant contribution $(B(1)=0.17 ; p=.002)$ to non-compliance, with age also making a significant contribution $(B(1)=-0.08 ; p=.02)$. A further analysis revealed that there was an interaction between reactance and treatment being perceived as a threat to freedom which contributed significantly to compliance $(B(1)=0.005 ; p=.002)$.

\section{Discussion}

The main hypothesis that highly reactant patients would be more likely to be noncompliant than patients exhibiting a low level of reactance was confirmed, although this finding was only apparent for past compliance. This is in accord with previous 
research involving patients with minor psychiatric disorders which demonstrated a greater degree of non-compliance in reactance-prone individuals compared with reactance individuals (Rohrbaugh, Tennen, Press, \& White, 1981; Graybar, Antonuccio, Boutilier, \& Varble, 1989).

Also consistent with previous research, age predicted past compliance, younger patients being less likely to be compliant (Tunnicliffe et al., 1992; Zito et al., 1985). Lack of family support did not significantly predict past compliance, but it did differentiate between current compliers and non-compliers.

An interaction between reactance and perceived threat from treatment provision also predicted past compliance. Brehm (1966) suggested that the magnitude of reactance depends in part upon the importance of the freedom that is being threatened. In the present study, reactance prone individuals who perceived treatment provision to be a threat to their freedom of choice were the most likely to have been non-compliant. Patients suffering from schizophrenia may be offered few choices in treatment other than medication, as this represents the only form of treatment accepted widely. It may be that individuals who object strongly to their freedom of choice being threatened in this way are most likely to exhibit reactance, thus resulting in non-compliant behaviour.

Past compliance was found to be the best predictor of current compliance, in accord with previous research (Buchanan, 1992). An unpleasant changed subjective state as measured by the DAI also predicted current compliance, although to a lesser degree. It is apparent in the present study that poor subjective response is more important than reactance in determining whether an individual is currently compliant or not. This finding provides supportive evidence for previous research which has demonstrated that poor subjective responses are associated with non-compliance (Awad, 1993; Naber, Walther, \& Kirchere, 1994).

Reactance was not found to predict current compliance. The observation that there were no significant differences in reactance between current compliers and noncompliers suggests that highly reactant individuals who have been non-compliant in the past may now be compliant, even though they are still highly reactant. This change in behaviour may be due to an improvement in subjective response or insight, or may be due to a decrement in the degree of perceived threat that treatment provision poses to reactant individuals. Clinical experience suggests that over time patients learn that their medication is useful to them in preventing relapse.

The results of the present study should be interpreted with some caution as current non-compliers were more difficult to recruit for the study. Patients were reporting on their own compliance level, which may have been over reported, particularly with regard to current compliance. In addition, patients may have been more willing to report past non-compliance as opposed to current non-compliance. A further limitation of the study involves the assessment of past and current compliance. Ideally, newly diagnosed patients would be assessed and followed-up using some more objective method of assessing compliance, for example pill counting, or the proportion of prescribed depot injections missed over a 9-month period. A further consideration is the validity of analysing the relationships between present scores on the HPRS, PRS, DAI and past compliance. This is a further agreement for a prospective study where data could be collected at two or more points in time. It 
would also be important to use a standardized measure of the perceived freedom of choice posed by treatment provision.

This study offers two variables not considered previously in research aimed at predicting non-compliance with anti-psychotic medication. Reactance and the perceived freedom of choice posed by treatment provision may be valuable in predicting future risk of non-compliance. Furthermore, they may be useful in informing psychological interventions aiming to increase compliance. Noncompliant individuals who exhibit a high reactance level, and perceive treatment provision to be a threat to their personal freedom might be targeted in order to achieve this. These variables might be used to select newly diagnosed individuals and to ensure that they receive a type of treatment provision that reduces the likelihood of inducing reactance, and increases the probability of perceiving treatment as beneficial. This corresponds with the suggestion that treatment provision will be most effective when the patients' viewpoint is taken into account, particularly in individuals who are concerned with autonomy and self-control (Day, Bentall, \& Warner, 1996). A key aspect of motivational interviewing is to avoid persuasion and enhance the patient's view that they are responsible for treatment decisions (Miller \& Rollnick, 1991). The present findings may explain why a new approach to the engagement of patients suffering from schizophrenia, based on motivational interviewing, has been shown to enhance compliance and improve outcome in the long term (Kemp et al., 1996).

\section{References}

Awad, A. G. (1993). Subjective response to neuroleptics in schizophrenia. Schizophrenia Bulletin 19, $609-616$.

Baekeland, F., \& Lundwall, L. (1975). Dropping out of treatment: A critical review. Psychological Bulletin, 82, 735-783.

Barrowclough, C., Tarrier, N., Lewis, S., Sellwood, W., Mainwaring, J., Quinn, J., \& Hamlin, C. (1999). A randomised controlled effectiveness trial of a needs based psychosocial intervention service for carers of schizophrenia patients. British Journal of Psychiatry, 174, 505-511.

Bebbington, P., \& Kuipers, L. (1994). The predictive utility of expressed emotion in schizophrenia: An aggregate analysis. Psychological Medicine, 24, 707-718.

Birchwood, M., Smith, J., Drury, V., Healy, J., Macmillan, F., \& Slade, M. (1994). A self-report insight scale for psychosis: Reliability, validity, and sensitivity to change. Acta Psychiatrica Scandinavica, 89, 62-67.

Brehm, J. W. (1966). A theory of psychological reactance. New York: Academic Press.

Brehm, J. W., \& Brehm, S. S. (1981). Psychological reactance. New York: Wiley.

Buchanan, A. (1992). A two-year prospective study of treatment compliance in patients with schizophrenia. Psychological Medicine, 22, 787-797.

Day, J. C., Bentall, R. P., \& Warner, S. (1996). Schizophrenic patients' experiences of neuroleptic medication: A Q-methodological investigation. Acta Psychiatrica Scandinavica, 93, 397-402.

Dowd, E. T., Milne, C. R., \& Wise, S. L. (1991). The Therapeutic Reactance Scale: A measure of psychological reactance. Journal of Counselling and Development, 69, 541-545.

Fleischhacker, W. W., Meise, U., Gunther, V., \& Kurz, M. (1994). Compliance with antipsychotic drug treatment: Influence of side effects. Acta Psychiatrica Scandinavica 89, (Suppl. 382), 11-15.

Ghaemi, S. N., \& Pope, H. G. (1994). Lack of insight in psychotic and affective disorders: A review of empirical studies. Harvard Review of Psychiatry, 2, 22-33.

Graybar, S. R., Antonuccio, D. O., Boutilier, L. R. \& Varble, D. L. (1989). Psychological reactance as a factor affecting patient compliance to physician advice. Scandinavian Journal of Behavioural Therapy, 18, 43-51. 
Hogan, T. P., Awad, A. G., \& Eastwood, M. R. (1983). A self-report scale predictive of drug compliance in schizophrenics: Reliability and discriminative validity. Psychological Medicine, 13, 177-183.

Hoge, S. K., Applebaum, P.S., Lawlor, T., Beck, J. C., Litman, R., Greer, A., Gutheil, T. G., \& Kaplan, E. A. (1990). Prospective multi-centre study of patients' refusal of antipsychotic medication. A rchives of General Psychiatry, 47, 949-956.

Hong, S. M., \& Page, S. (1989). A psychological reactance scale: Development, factor structure and reliability. Psychological Reports 64, 1323-1326.

Kasper, J. A., Hoge, S. K., Feucht-Haviar, T., Cortina, J., \& Cohen, B. (1997). Prospective study of patients' refusal of antipsychotic medication under a physician discretion review procedure. American Journal of Psychiatry, 154, 483-489.

Kelly, G. R., Mamon, J. A., \& Scott, J. E. (1987). Utility of the health belief model in examining medication compliance among psychiatric out-patients. Social Sciences and Medicine, 25, 1205-1211.

Kemp, R., David, A., \& Hayward, P. (1996). Compliance therapy: An intervention targeting insight and treatment adherence in psychotic patients. Behavioural and Cognitive Psychotherapy, 24, 331-350.

Krawiecka, M., Goldberg, D., \& Vaughan, M. (1977). A standardised psychiatric assessment scale for rating chronic psychotic patients. Acta Psychiatrica Scandinavica, 55, 299-308.

Miller, W. R., \& Rollnick, S. (1991). Motivational interviewing: Preparing people to change addictive behaviour. New York: Guilford.

Naber, D., Walther, A., \& Kirchere, T. (1994). Subjective effects of neuroleptics predict compliance. In W. Gaebel \& A. G. Awad (Eds.), Prediction of neuroleptic treatment outcome in schizophrenia: Concepts and methods (pp. 111-122). Heidelberg: Springer-Verlag.

Rohrbaugh, M., Tennen, H., Press, S., \& White, L. (1981). Compliance, defiance, and therapeutic paradox: Guidelines for strategic use of paradoxical interventions. A merican Journal of Orthopsychiatry, 51, 454-467.

Tunnicliffe, S., Harrison, G., \& Standen, P. J. (1992). Factors affecting compliance with depot injection treatment in the community. Social Psychiatry and Psychiatric Epidemiology, 27, 230-233.

Vaughn, C. E., \& Leff, J. P. (1976). The influence of family and social factors on the course of psychiatric illness: A comparison of schizophrenic and depressed neurotic patients. British Journal of Psychiatry, 129, 125-137.

World Health Organization. (1978). Mental disorders: Glossary and guide to their classification in accordance with the ninth revision of the International Classification of Diseases (ICD-9). Geneva: World Health Organization.

Zito, J. L., Routt, W. W., Mitchell, J. E., \& Roerig, J. L. (1985). Clinical characteristics of hospitalised psychotic patients who refuse antipsychotic drug therapy. American Journal of Psychiatry, 142, $822-826$. 